\title{
Regulation of dopamine release by CASK- $\beta$ modulates locomotor initiation in Drosophila melanogaster
}

\author{
Justin B. Slawson ${ }^{\dagger}$, Elena A. Kuklin, Konark Mukherjee ${ }^{\dagger}$, Nicolás Pírez $^{\dagger}$, Nathan C. Donelson ${ }^{\dagger}$ \\ and Leslie C. Griffith*
}

Department of Biology, Volen Center for Complex Systems, National Center for Behavioral Genomics, Brandeis University, Waltham, MA, USA

Edited by:

Martin Giurfa, Centre National de la Recherche Scientifique - Université Paul Sabatier-Toulouse III, France

Reviewed by:

James J. L. Hodge, University of Bristol, UK

David Evan Krantz, UCLA, USA

*Correspondence:

Leslie C. Griffith, Department of

Biology, Volen Center for Complex

Systems, National Center for

Behavioral Genomics, Brandeis

University, MS008, 415 South St.,

Waltham, MA 02454, USA

e-mail: griffith@brandeis.edu

\section{${ }^{\dagger}$ Present address:}

Justin B. Slawson and Nathan C.

Donelson, Biogen Idec Inc., 14

Cambridge Center, Cambridge, MA

02142, USA;

Konark Mukherjee, Virginia Tech

Carilion Research Institute, Virginia

Tech Biological Sciences, 2

Riverside Cir., B2003, Roanoke, VA

24016, USA;

Nicolás Pírez, Fundación Instituto

Leloir and IIBBA-CONICET, Av.

Patricias Argentinas 435

(1405-BWE), Buenos Aires,

Argentina
CASK is an evolutionarily conserved scaffolding protein that has roles in many cell types. In Drosophila, loss of the entire CASK gene or just the CASK- $\beta$ transcript causes a complex set of adult locomotor defects. In this study, we show that the motor initiation component of this phenotype is due to loss of CASK- $\beta$ in dopaminergic neurons and can be specifically rescued by expression of CASK- $\beta$ within this subset of neurons. Functional imaging demonstrates that mutation of $C A S K-\beta$ disrupts coupling of neuronal activity to vesicle fusion. Consistent with this, locomotor initiation can be rescued by artificially driving activity in dopaminergic neurons. The molecular mechanism underlying this role of CASK- $\beta$ in dopaminergic neurons involves interaction with Hsc70-4, a molecular chaperone previously shown to regulate calcium-dependent vesicle fusion. These data suggest that there is a novel CASK- $\beta$-dependent regulatory complex in dopaminergic neurons that serves to link activity and neurotransmitter release.

Keywords: Drosophila melanogaster, neurotransmitter release, CASK, dopamine, locomotion, Hsc70-4

\section{INTRODUCTION}

Dopamine is a biogenic amine present in most nervous systems. Its neuromodulatory function is highly conserved across species, contributing to complex biological states and behaviors, including locomotion, attention, learning, sleep, and mood (Costa, 2007; Palmiter, 2008). Not surprisingly, misregulation of dopaminergic signaling can lead to the development of a number of different human pathologies, the most common of which is Parkinson's Disease, a neurodegenerative condition characterized by the progressive loss of dopaminergic cells within the substantia nigra (Braak and Del Tredici, 2008). Loss of dopaminergic cells has been directly linked to aberrations in motor function, including tremor, rigidity, bradykinesia, and postural instability (Lees et al., 2009). Attempts to characterize the disease at the cellular level have suggested that excitotoxicity, mitochondrial dysfunction, oxidative stress, and protein misfolding/aggregation are all important pathophysiological features (Schapira, 2009; Vaarmann et al., 2013), but the primary cause of the disease has remained elusive (Obeso et al., 2010). The vast majority of cases are idiopathic and may stem from heightened sensitivity of the dopaminergic system to inflammation and environmental toxins/stressors, possibly in conjunction with unidentified genetic factors (Thomas and Beal, 2007; Surmeier et al., 2010).

One interesting feature of the pathology observed in Parkinson's disease is that despite the fact that the entire brain is subjected to the same genetic and environmental factors, only the dopaminergic neurons appear to be especially vulnerable. For this reason, it has been suggested that there are likely to be cell-specific risk factors that predispose dopaminergic cells to dysfunction (Obeso et al., 2010; Surmeier et al., 2010). Despite this, however, little work has been done to elucidate differences between dopaminergic cells and other neurons that are relevant to this phenomenon.

The function of dopamine in locomotion is highly conserved across species, and insects also show a Parkinsonian-like motor initiation deficit upon disruption of this neurotransmitter system (Feany and Bender, 2000; Riemensperger et al., 2011). In this study, we demonstrate that CASK- $\beta$, a Drosophila scaffolding 
protein orthologous to mammalian CASK, regulates motor initiation by facilitating transmitter release specifically in dopaminergic cells. Previous studies have implicated CASK- $\beta$ in proper locomotor behavior (Martin and Ollo, 1996; Sun et al., 2009; Slawson et al., 2011). The severity of the motor phenotype resulting from loss of CASK- $\beta$ is striking, and appears to influence several different locomotor parameters, including motor initiation, motor maintenance, speed, and acceleration. Recent work (Slawson et al., 2011) mapped the spatial requirement for CASK- $\beta$ to a discrete subset of cells within the fly central nervous system that did not include major known premotor neuron groups.

Using directed expression via the GAL4/UAS system (Fischer et al., 1988; Brand and Perrimon, 1993; Phelps and Brand, 1998), we show here that a number of cells within this subset are dopaminergic neurons, and that reconstitution of CASK- $\beta$ specifically in these cells selectively rescues the motor initiation component of the mutant's complex locomotor phenotype. The motor initiation disruption in CASK- $\beta$ mutants likely stems from aberrations in vesicle release at dopaminergic synapses, which may be driven by a recently identified interaction between CASK$\beta$ and the molecular chaperone Hsc70-4 (Mukherjee et al., 2014). Interestingly, while this interaction appears to be present in multiple cell types throughout the invertebrate nervous system, our results indicate that the association between these two proteins is only behaviorally relevant to locomotion in dopaminergic cells. Hsc70-4 and its homologs have been implicated in mammalian models of Parkinson's disease (Auluck et al., 2002; Pemberton et al., 2011) and their levels are altered in brain samples from human patients (Alvarez-Erviti et al., 2010; Sala et al., 2014). Whether regulation of dopamine release by an Hsc4/CASK interaction is relevant to mammalian dopaminergic function is unknown, but our results suggest that this may be an interesting new avenue of inquiry.

\section{MATERIALS AND METHODS FLY STOCKS AND MAINTENANCE}

All flies were raised at $25^{\circ} \mathrm{C}$ in a $12 \mathrm{~h}: 12 \mathrm{~h}$ light:dark cycle unless otherwise stated. Flies were fed standard cornmeal-dextrose agar media. UAS-CASK- $\beta$-YFP and its domain deletions were subcloned into EcoRI/XhoI sites of a PhiC31 vector. DNA was sent to Rainbow Transgenic Flies, Inc. (Camarillo, CA, USA) for embryonic injections into fly strain \#24872 (Genotype: M\{vasint.Dm $\} Z H-2 A, P B a c\{y[+]-a t t P-3 B\} 8 V K 00037)$ to produce flies with targeted insertions on the second chromosome. Transformants were selected by eye color based on the presence of the mini-white gene. All other fly lines were obtained from the Bloomington Stock Center (Bloomington, IN, USA), except for TH-Gal4 (from Jay Hirsh, University of Virginia, Charlottesville, USA), C164-Gal4 (from Vivian Budnik, University of Massachusetts, Worcester, MA, USA), dilp2-Gal4 (from Ulrike Heberlein, Janelia Farms), TH-Gal80 (from Julie Simpson, Janelia Farms), UAS-dTRPA1 (from Paul Garrity, Brandeis University, Waltham, USA), UAS-synaptopHluorin (from Gero Miesenböck, University of Oxford, UK), Hsc70-4 $\Delta^{19}$ (a strong hypomorphic allele from Konrad Zinsmaier, University of Arizona, Tucson, AZ, USA), $t u b-G A L 80^{\text {ts }}$ (Ron Davis, Scripps Florida) and the fulllength UAS-CASK- $\beta$ line, which was UAS-CASK 10.20 (from
Peter Bryant, University of California, Irvine, CA, USA). For behavioral rescue experiments, all GAL4 and UAS- lines were crossed into the CASK- $\beta^{\mathrm{P} 18}$ null background (Slawson et al., 2011 ) to ensure that endogenous protein wouldn't confound the tissue-specific reconstitution of CASK- $\beta$.

\section{P-ELEMENT MOBILIZATION}

The previously described TH-Gal4 line (Friggi-Grelin et al., 2003) demonstrated locomotor abnormalities without the addition of any GAL4-responsive transgenes (data not shown). As this could make it difficult to properly quantify changes in movement following the reconstitution of CASK, we utilized $P$-element excision to mobilize the TH-Gal4 construct to a new chromosomal location, which we have named THumpGal4. To generate THump-Gal4, the third chromosome THGal4 P-element construct was mobilized using standard genetic techniques (Greenspan, 1997). Briefly, TH-Gal4 flies were crossed with w;+/CyO $\Delta 2-3$;+/TM6B flies. F1 male progeny were mated with $\operatorname{Df}(1) \mathrm{w}$ females, and the resulting F2 progeny were selected for $P$-element mobilization based on retention of both eye color and the humoral trait associated with TM6B. The resulting insertion was mapped to the second chromosome, and expresses GAL4 in the same sets of dopaminergic cells observed in the original TH-Gal4 line (see Supplemental Figure S1).

\section{CONFOCAL IMAGING}

Adult brains were dissected in phosphate buffered saline (PBS), fixed for $15 \mathrm{~min}$ in $4 \%$ paraformaldehyde, washed in PBT $(0.5 \%$ Triton X-100 in PBS), stained in primary antibodies at $4^{\circ} \mathrm{C}$ for ca. $48 \mathrm{~h}$, washed in PBT, stained in fluorescent secondary antibodies at $4^{\circ} \mathrm{C}$ for overnight, then washed in PBT and mounted using Vectashield (Vector Laboratories, Inc.). A Leica TCS SP5 confocal microscope was used to capture sequential images of the brains at $20 \times$ magnification with LAS AF imaging software from Leica (version 2.6.0). Anti-TH mouse monoclonal antibody used in these experiments was obtained from Immunostar (Hudson, WI, USA), and was used at a concentration of 1:500. AntiGFP rabbit polyclonal antibody was procured from Santa Cruz Biotechnology, Inc., and was also used at a concentration of 1:500. Alexa633 anti-mouse antibody and alexa488 anti-rabbit antibody (Invitrogen) were both used as secondary antibodies at 1:200. All antibodies were diluted in PBT $+10 \%$ normal goat serum (NGS Jackson ImmunoResearch Laboratories).

\section{LOCOMOTOR ANALYSIS}

High-resolution video tracking was performed as described (Slawson et al., 2009, 2011). Briefly, mated male flies aged 13 days were sorted into groups of 10 under light $\mathrm{CO}_{2}$ anesthesia. Following at least a $48 \mathrm{~h}$ recovery at $25^{\circ} \mathrm{C}$, flies at $4-6$ days of age were gently knocked into a translucent observation chamber. Following a $30 \mathrm{~min}$ acclimation period, the trial was initiated by the administration of a brief pulse of air, and fly movement was video-recorded for $30 \mathrm{~s}$. Ten seconds prior to the administration of the air pulse, the chambers were given five gentle taps on a padded surface to wake the flies up for testing. Locomotor traces of individual flies were recorded using DIAS 3.2 software (Soll, 1995; Soll et al., 2001) and analyzed using 
custom-designed scripts in Matlab (Mathworks, Natick, MA, USA). In the behavioral rescue experiments, all measurements for UAS- or Gal4- containing lines were normalized to control flies by dividing each individual average by the mean of wild type flies for a given parameter. In these cases, wild type performance for each parameter is denoted by a dotted black line in the bar graphs. At least seven trials over three or more days were performed for each genotype. For experiments using dTRPA1, flies were grown and maintained at $23^{\circ} \mathrm{C}$. Three hours before behavioral testing, flies were shifted to $27^{\circ} \mathrm{C}$ and subsequently assayed at this temperature. For experiments utilizing $\mathrm{Gal} 80^{\text {ts }}$, flies were grown and maintained at $18^{\circ} \mathrm{C}$ until sorting for behavioral analysis. During the $48 \mathrm{~h}$ recovery window preceding behavioral testing, flies were shifted to $25^{\circ} \mathrm{C}$ and assayed at this temperature. In all cases, locomotor chambers were pre-equilibrated to the testing temperature for at least $30 \mathrm{~min}$.

\section{SYNAPTOPHLUORIN IMAGING}

Imaging experiments were performed using a naked brain preparation. Briefly, fly brains are dissected out of the head of the animal and placed in a perfusion chamber (Harvard Apparatus) immediately before the experiments. Brains were allowed to recover in the perfusion chamber before recording started. The preparation was under constant perfusion with artificial hemolymph-like (AHL) saline flowing by gravity feed at approximately 3-4 ml/min. The AHL contains $5 \mathrm{mM}$ HEPES, $4 \mathrm{mM}$ $\mathrm{NaHCO}_{3}$ pH 7.5, $108 \mathrm{mM} \mathrm{NaCl}, 5 \mathrm{mM} \mathrm{KCl}, 2 \mathrm{mM} \mathrm{CaCl}, 8.2$ $\mathrm{mM} \mathrm{MgCl} 2,1 \mathrm{mM} \mathrm{NaH} \mathrm{PO}_{4}, 5 \mathrm{mM}$ Trehalose, $10 \mathrm{mM}$ sucrose (Wang et al., 2003). We recorded each brain for a period of $240 \mathrm{~s}$ ( $2 \mathrm{~Hz}$ frequency). Brains were stimulated by perfusion of $5 \mathrm{mM}$ carbachol (carbamoylcholine chloride, Sigma) for $30 \mathrm{~s}$ by switching between reservoirs with a manually controlled 3way solenoid. All experiments were performed using an Olympus BX51WI microscope and a $60 \times(0.9$ NA) water immersion lens (Olympus LUMPlanFl), using the following filter set: Exciter HQ470/40×, Dichroic Q495LP, Emitter HQ525/50 m. The acquisition was performed using the open-source software $\mu$ Manager (Edelstein et al., 2010), and recordings are saved using a CCD camera (Hammamatsu Orca C472-80-12AG). Data were analyzed offline using custom software written in Image (NIH), Matlab (Mathworks) and Excel (Microsoft).

\section{IMMUNOBLOTS}

Heads from frozen male and female flies were removed by vortexing in liquid nitrogen and homogenized in $1 \times$ SDS buffer. Protein samples were separated by SDS-PAGE and transferred to nitrocellulose. Immunoblots were visualized using either ECL or Luminol detection reagents (GE Healthcare) on film (developed with a Kodak X-OMAT 200A Developer). The polyclonal antibody used to visualize CASK (Marble et al., 2005) was a kind gift from Gisela Wilson (University of Wisconsin, Madison, WI, USA). Green fluorescent protein and actin monoclonal antibodies were obtained commercially (Roche and Millipore, respectively). All primary antibodies were used at a concentration of 1:1000. Horseradish peroxidase-conjugated anti-guinea pig (Jackson Laboratories) and anti-mouse (GE healthcare) secondary antibodies were used at a concentration of 1:5000.

\section{STATISTICS}

All statistical analysis was done using either JMP software (SAS Institute, Cary, NC, USA) or Microsoft Excel. For all imaging experiments, significance was determined using a two-tailed Student's $t$-test $(P<0.05)$. For all behavioral manipulations, one-way analysis of variance (ANOVA) was computed, and a Tukey HSD (honest significance difference) test was used for post hoc analysis as previously reported (Slawson et al., 2011). Error bars in all figures represent the standard error of the mean (SEM), with significant differences between groups indicated by different letters (conditions that are not statistically different from one another are labeled with the same letter) or asterisks $(\alpha<0.05)$.

\section{RESULTS EXPRESSION OF CASK- $\beta$ IN DOPAMINERGIC NEURONS RESCUES MOTOR INITIATION DEFECTS}

Expression of CASK- $\beta$ under control of the C164-Gal4 driver line can rescue motor behavior in CASK- $\beta$ mutants (Slawson et al., 2011). This driver line expresses in a discrete subset of CNS neurons, many of which are difficult to identify based on morphology and position alone. None of the known motor control centers, including central complex regions (Strauss and Heisenberg, 1993; Martin et al., 1999; Strauss, 2002) and mushroom bodies (Martin et al., 1998) are strongly represented in the C164-Gal4 pattern (Slawson et al., 2011). Although it is interesting that these brain regions are not a direct site of action for CASK- $\beta$ in locomotion, this does not rule out the possibility that CASK- $\beta$ affects the function of one of these known motor centers indirectly. One way that this could occur is if CASK acted in modulatory dopaminergic neurons, which have been implicated in a number of motor disorders (reviewed in Piccini, 2004), and also directly innervate the central complex (Kong et al., 2010; White et al., 2010) and mushroom bodies (Neckameyer, 1998; Mao and Davis, 2009; White et al., 2010). Indeed, dissecting the expression pattern of C164-Gal4 revealed the presence of dopaminergic neurons from several different cell clusters (Figure 1). Intriguingly, a number of the dopaminergic cells identified map to the PPL1 cluster (Figures 1B,C), which is an important site of degeneration in many fly models of Parkinson's Disease which share similar locomotor abnormalities (Whitworth et al., 2005; Trinh et al., 2008).

To directly assess the hypothesis that dopaminergic cells are involved in the CASK- $\beta$ locomotor phenotype, we attempted to rescue the behavior in a null animal by reconstituting CASK- $\beta$ specifically in these neurons using the GAL4/UAS binary expression system (Fischer et al., 1988; Brand and Perrimon, 1993; Phelps and Brand, 1998). Targeted expression within these cells was achieved through use of a new GAL4 line called THumpGal4, which drives expression specifically in tyrosine hydroxylasepositive cells (Supplemental Figure S1). Interestingly, expression of CASK- $\beta$ with THump-Gal4 significantly rescued motor behavior, but only in the "Pause Length" parameter, which is a measure of motor initiation. The other three behavioral metrics previously shown to be affected in the null animals (Slawson et al., 2011) remained unchanged (Figure 2A). In addition, ectopic expression of CASK- $\beta$ with the C164-Gal4 driver line failed to 

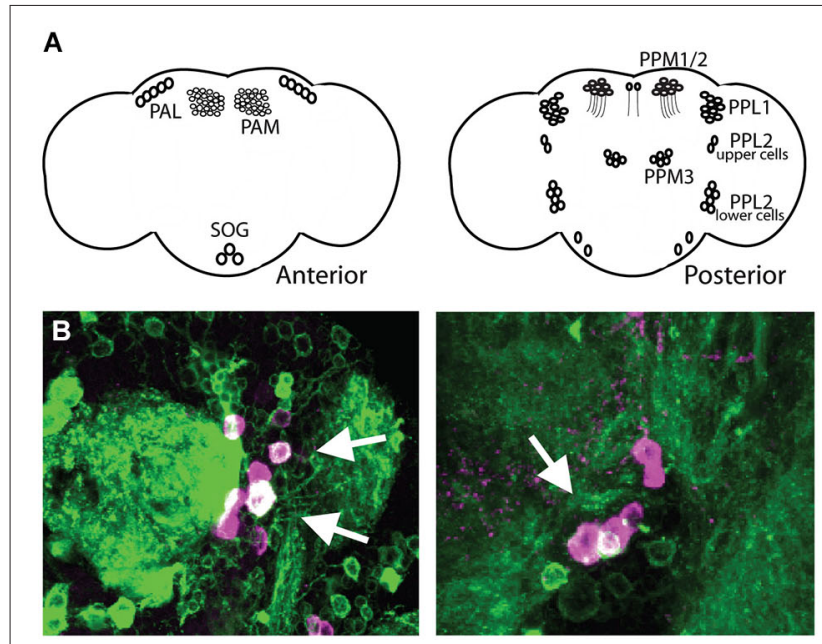

PPL1

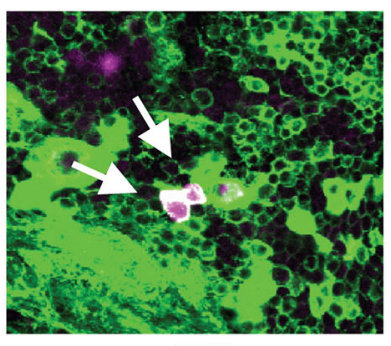

PPM3

PPL2 lower

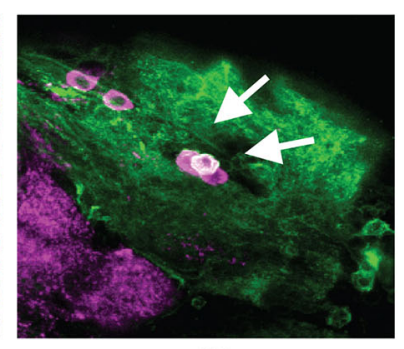

PAL

C

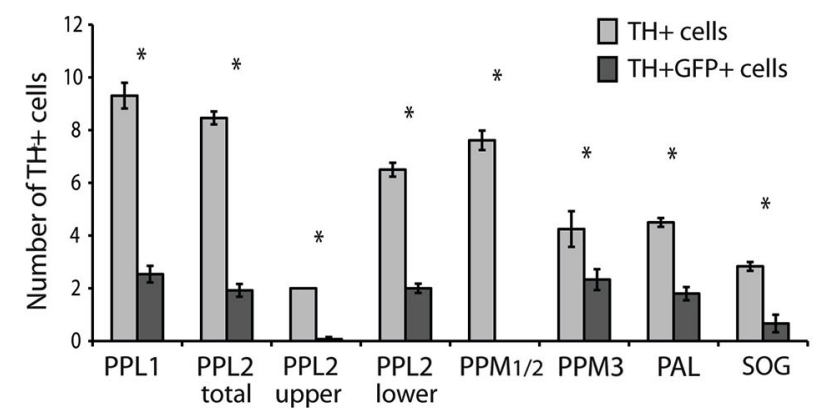

FIGURE 1 | C164-Gal4 expresses in dopaminergic neurons.

(A) Dopaminergic neurons in the Drosophila brain are organized into distinct clusters (Mao and Davis, 2009). Left panel shows anterior brain clusters. Right panel shows clusters in the posterior brain. (B) The C164-Gal4 expression pattern visualized by crossing the GAL4 line to UAS-mCD8GFP. Brains were co-stained with anti-TH (shown in purple) to reveal dopaminergic neurons, while visualization of GFP expression was enhanced with an anti-GFP antibody (shown in green). The two staining patterns are presented as an overlay, and the arrows indicate points of overlap between the two expression patterns. (C) The number of TH+ and TH+GFP+ double positive cells in each dopaminergic neuron cluster were counted. The C164-Gal4 driver expresses in specific cells within the PAL, PPL1, PPL2, and PPM3 clusters. Significant differences between $\mathrm{TH}+$ and TH+GFP+ double positive staining are represented by asterisks $(P<0.05$, Student's $t$-test). See Section Methods for details on statistical tests.

rescue the pause length parameter when dopaminergic expression was simultaneously repressed using TH-Gal80 (Supplemental Figure S2). These results indicate that individual components of the locomotor phenotype are the result of CASK- $\beta$ deficiency in different neuroanatomical loci within the C164-GAL4 ${ }^{+}$neurons of the central nervous system, suggesting that these are independently controlled behavioral parameters.

Abnormalities in dopaminergic signaling have been shown to produce similar motor initiation deficits in the fly (Riemensperger et al., 2011), and degeneration of dopamine-producing cells is believed to underlie locomotor abnormalities in Drosophila models of Parkinson's Disease (reviewed in Muñoz-Soriano and Paricio, 2011). For this reason, we assessed the dopaminergic system of wild type and CASK- $\beta$ mutants to see if there were any significant differences in cell numbers. Interestingly, none of the dopaminergic cell groups showed significant reductions in the mutant (Figures $2 \mathbf{B}, \mathbf{C}$ ). In contrast, loss of CASK- $\beta$ led to a small, but significant increase in $\mathrm{TH}^{+}$cells within the PPL2 cluster (Figure 2C). This implies that the motor initiation phenotype does not stem from loss of dopaminergic cells. The cause for the small increase in $\mathrm{TH}^{+}$cells in the mutant is unknown, but could be due to differences in genetic background or a role for CASK- $\beta$ in repression of TH expression in a small population of neurons.

\section{CASK- $\beta$ MUTANTS DEMONSTRATE A FUNCTIONAL DEFICIT IN DOPAMINE RELEASE}

Previous work at the larval neuromuscular junction has suggested that Drosophila CASK affects locomotor behavior through modulation of synaptic vesicle release (Zordan et al., 2005; Chen and Featherstone, 2011), most probably by altering calcium responsiveness (Gillespie and Hodge, 2013). To address the issue of whether or not synaptic vesicle release underlies the adult dopaminergic locomotor phenotype, we performed optical imaging experiments using flies that express the $\mathrm{pH}$-sensitive sensor synaptopHluorin $(\mathrm{SpH})$ under control of THump-Gal4. This sensor reports synaptic vesicle fusion to the membrane by means of a membrane bound $\mathrm{pH}$-sensitive variant of GFP (Miesenböck et al., 1998; Ng et al., 2002). SynaptopHluorin is quenched in the acidic environment of the vesicular lumen, while highly fluorescent in the higher $\mathrm{pH}$ encountered within the synaptic cleft (Miesenböck et al., 1998). Changes in fluorescence thus act as a measure of vesicle release following synaptic activity or stimulation. For these experiments, we expressed $\mathrm{SpH}$ in dopaminergic cells of either a wild type or $C A S K-\beta$ null animal and measured changes in fluorescence within a glomerulus of the antennal lobe (a region with dense dopaminergic innervation) using a naked brain preparation (Figure 3A). Since acetylcholine is the major excitatory neurotransmitter in the insect brain (Leech and Sattelle, 1993), we stimulated neurons by applying $5 \mathrm{mM}$ carbachol, a cholinergic agonist. We found that the peak response elicited by carbachol was significantly decreased in the dopaminergic terminals of null flies compared with wild type brains (Figures 3B,C). This finding suggests that loss of CASK- $\beta$ results in a functional deficit of synaptic output at dopaminergic synapses.

To determine whether simply increasing the activity level of dopaminergic neurons was sufficient to rescue the locomotor deficit seen in these flies, we hyperactivated dopaminergic neurons using dTRPA1, a warmth-gated non-specific cation channel 


\section{A}
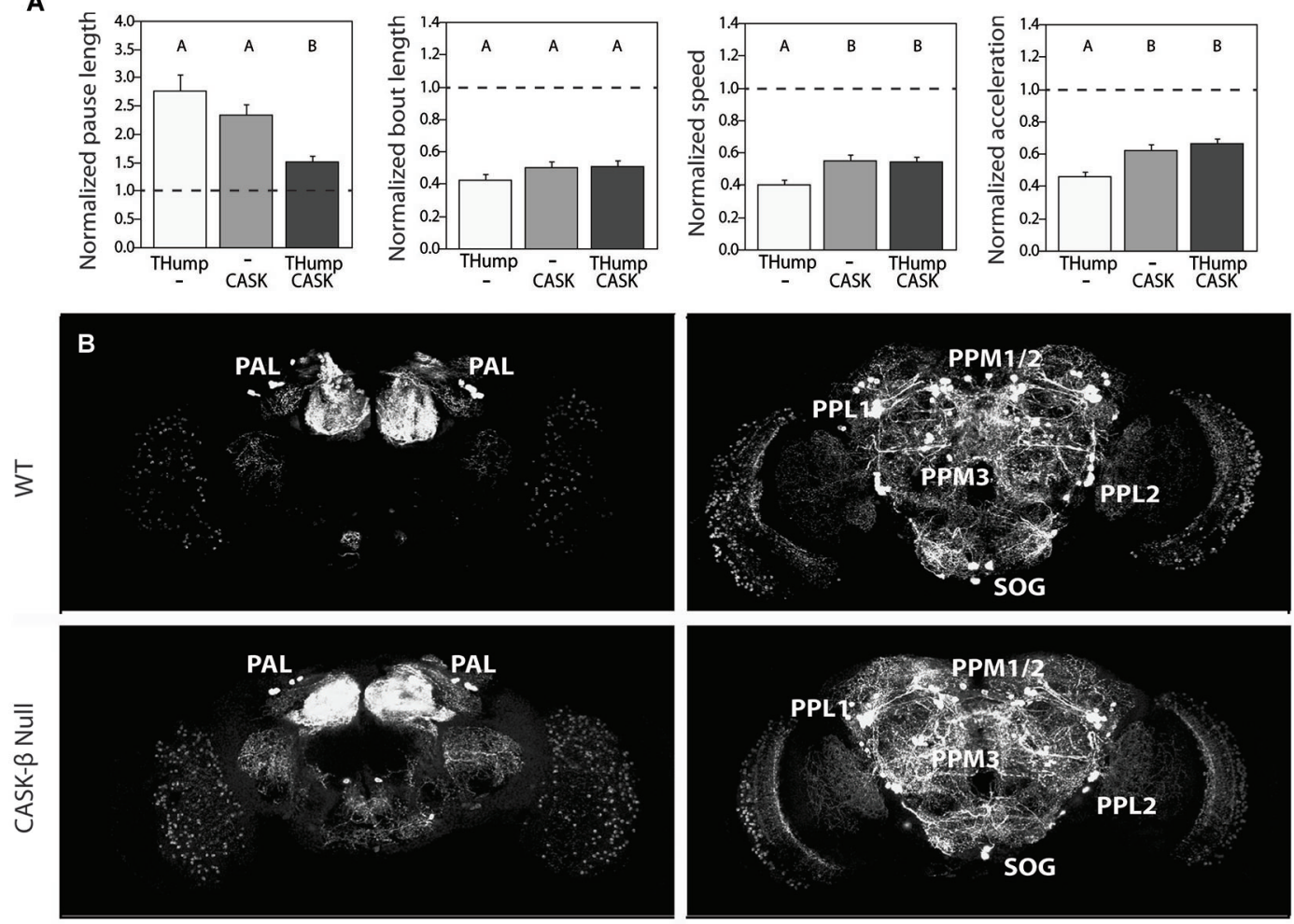

C

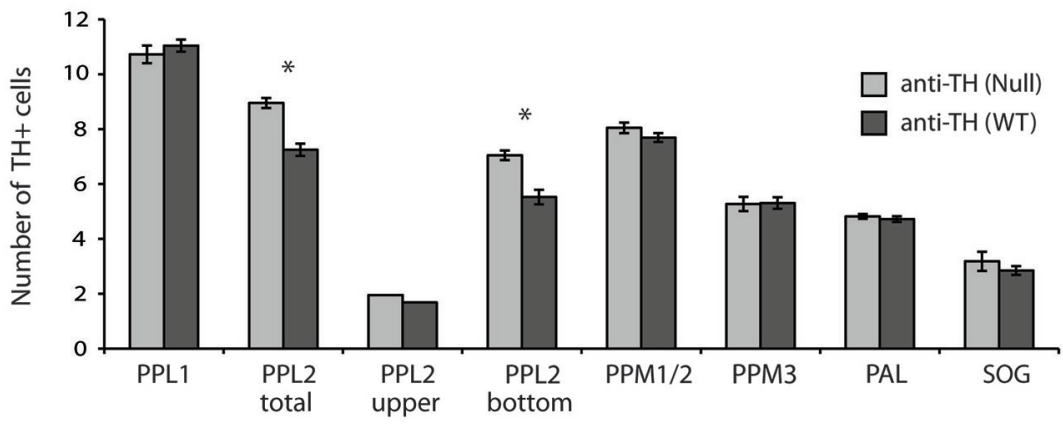

FIGURE 2 | CASK- $\beta$ contributes to dopaminergic control of motor initiation without disrupting the neuronal architecture of dopaminergic cell clusters. (A) Ectopic expression of CASK- $\beta$ in dopaminergic neurons using Thump-Gal4 significantly rescues the pause length parameter of locomotor behavior, which is a measure of motor initiation. The other metrics of locomotion remained unchanged. Data from all groups are normalized to WT control levels that are shown as a dotted black line at 1.0 on the Y-axis of each graph. The magnitude of the deviation from the dotted line is proportional to severity of phenotype. Transgenes present in each group of animals are indicated below the histogram bars: "THump" = THump-Gal4, and "CASK" = UAS-CASK- $\beta$. All transgenes were on a CASK- $\beta$ mutant background. Letters indicate significant differences between groups $(P<0.05$, ANOVA with Tukey HSD). See Section Methods for details on statistical tests. (B) Dopaminergic neurons were visualized with an anti-TH antibody using confocal imaging. (C) Cell counts of both WT brains (top panels, anterior and posterior are shown) and CASK- $\beta$ null brains (bottom panels, anterior and posterior are shown) demonstrate equivalent numbers of dopaminergic neurons across most dopaminergic cell clusters between these two genotypes. Significant differences between WT and CASK- $\beta$ mutant are represented by asterisks ( $P<0.05$, Student's $t$-test).
(Hamada et al., 2008). Activation of these channels in dopaminergic neurons of $C A S K-\beta$ null flies by increasing temperature significantly rescued the "Pause Length" parameter, but had little effect on the other three indices (Figure 3D). This suggests that the motor initiation deficit observed in the null flies stems largely from a reduction in synaptic output from dopaminergic cells. These data also indicate that the reduction in $\mathrm{SpH}$ signal amplitude likely results from a quantitative difference in vesicle release, as opposed to a lack of dopamine production, pointing to a problem in coupling of neuronal activity to vesicle fusion.

\section{INITIATION OF MOTOR BEHAVIOR IS DEPENDENT ON THE ISOFORM-SPECIFIC N-TERMINUS OF CASK- $\beta$}

Mammalian CASK appears to play a general role in vesicle release through a neurexin-dependent mechanism (Hata et al., 1996; 
A

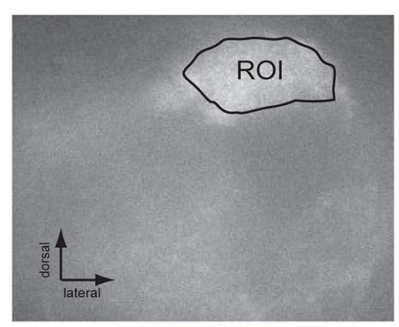

D

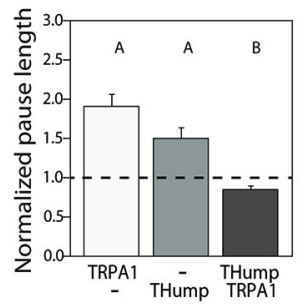

B
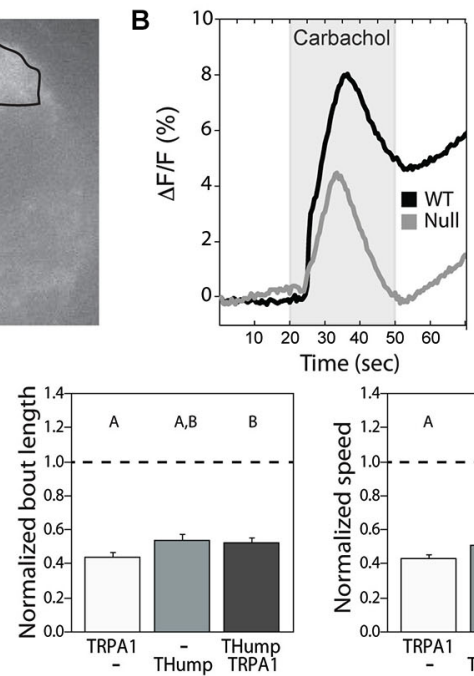

C

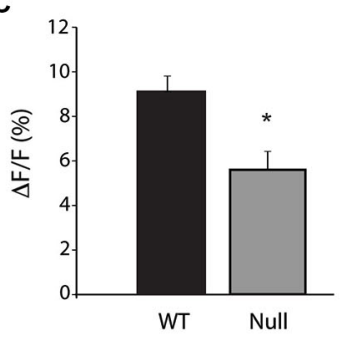

FIGURE 3 | Synaptic vesicle release deficits underlie the dopaminergic motor initiation phenotype. (A) SynaptopHluorin was used to visualize vesicle release from dopaminergic cells of the antennal lobes using a naked brain preparation. An example region of interest is shown. (B) Upon stimulation with carbachol, $C A S K-\beta$ mutants displayed a reduced synaptopHluorin response indicative of less vesicle fusion. (C) Average peak response was significantly different between WT and CASK $-\beta$ mutants ${ }^{*} P<0.05$, Student's $t$-test). (D) Increasing synaptic activity in dopaminergic neurons of CASK- $\beta$ mutant flies via dTRPA1 activation specifically rescued the pause length parameter of
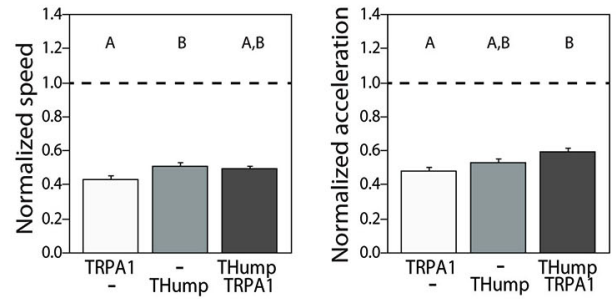

locomotion, but failed to cause a change in the other three metrics of motor behavior. Data from all groups are normalized to WT control levels which are shown as a dotted black line at 1.0 on the Y-axis of each graph. The magnitude of the deviation from the dotted line is proportional to severity of phenotype. Transgenes present in each group of animals are indicated below the histogram bars: "THump" = THump-Gal4, and "TRPA1" = UAS-dTRPA1. As above, all transgenes were on a CASK- $\beta$ mutant background. Letters indicate significant differences between groups ( $P<0.05$, ANOVA with Tukey HSD). See Section Methods for details on statistical tests.
Atasoy et al., 2007) and this has been posited to be important in Drosophila as well (Sun et al., 2009). In order to interact with neurexin, the N-terminal CaMK-like and L27 domains of mammalian CASK first bind with Mint1/X11 and Veli, respectively (Butz et al., 1998). This interaction is believed to modulate synaptic vesicle release in two ways. First, the N-terminal CaMKlike domain of CASK displays pseudokinase activity under certain conditions, and has been shown to phosphorylate neurexin in vivo (Mukherjee et al., 2008). Secondly, the tripartite complex of CASK, Mint1/X11, and Veli is capable of recruiting additional proteins to the presynaptic terminal, which indirectly influences neurexin-mediated exocytosis (Butz et al., 1998; Missler and Sudhof, 1998). For both of these mechanisms, the N-terminus of CASK is required for proper modulation of vesicle release from axon terminals.

Based on the high degree of homology between the N-terminal regions of mammalian CASK and Drosophila CASK- $\beta$, we set out to determine whether or not the CaMK-like and L27 domains are required for motor initiation in the fly. We generated three new YFP-tagged CASK- $\beta$ transgenes, each under the control of GAL4-responsive upstream activating sequence (UAS): two truncated forms (one missing the CaMK-like domain, the other without the L27 domains), and a full-length version (depicted in Figure 4A and visualized via immunoblot in Figure 4B). As expected, when expressed in dopaminergic cells, the full-length CASK- $\beta$-YFP significantly rescued motor initiation behavior as compared with mutant controls (Figure 4C). Both the CaMKlike and L27 deleted forms of CASK- $\beta$-YFP, however, failed to significantly rescue initiation (Figure 4C). While all three of these constructs express CASK- $\beta$, the $\Delta$ CaMK-like domain ablated line appears to have reduced expression, suggesting that this domain could also contribute to the overall stability of the protein (Figure 4B), but makes interpretation of the failure to rescue less clear. The expression level of the $\Delta$ L27 CASK protein, however, was similar to wild type, indicating that the L27 domains of the isoform-specific N-terminus are necessary for behavioral rescue. This is supportive of the idea that something analogous to the mammalian tripartite complex could be central to the role of CASK- $\beta$ in regulating output of dopaminergic neurons. Alternatively, it is possible that some other protein, also requiring interactions with the $\mathrm{N}$-terminal domain, is acting as a partner for CASK in regulation of dopaminergic neuron vesicle fusion. There are many candidates since CASK, as a member of the MAGUK family of scaffolding proteins, has been shown to have a multitude of binding partners (Zheng et al., 2011).

\section{CASK- $\beta$ INTERACTS GENETICALLY WITH Hsc70-4, A REGULATOR OF SYNAPTIC VESICLE RELEASE, TO REGULATE LOCOMOTION}

Because CASK has so many potential binding partners, it is important to have a methodology to identify putative cell-specific interactors. Recently, we developed a method for mass spectrometry identification of co-immunoprecipitated proteins bound to full length CASK- $\beta$-YFP expressed in defined neuronal populations using the GAL4/UAS system (Mukherjee et al., 2014). This published data set allowed us to obtain a list of cell-specific 

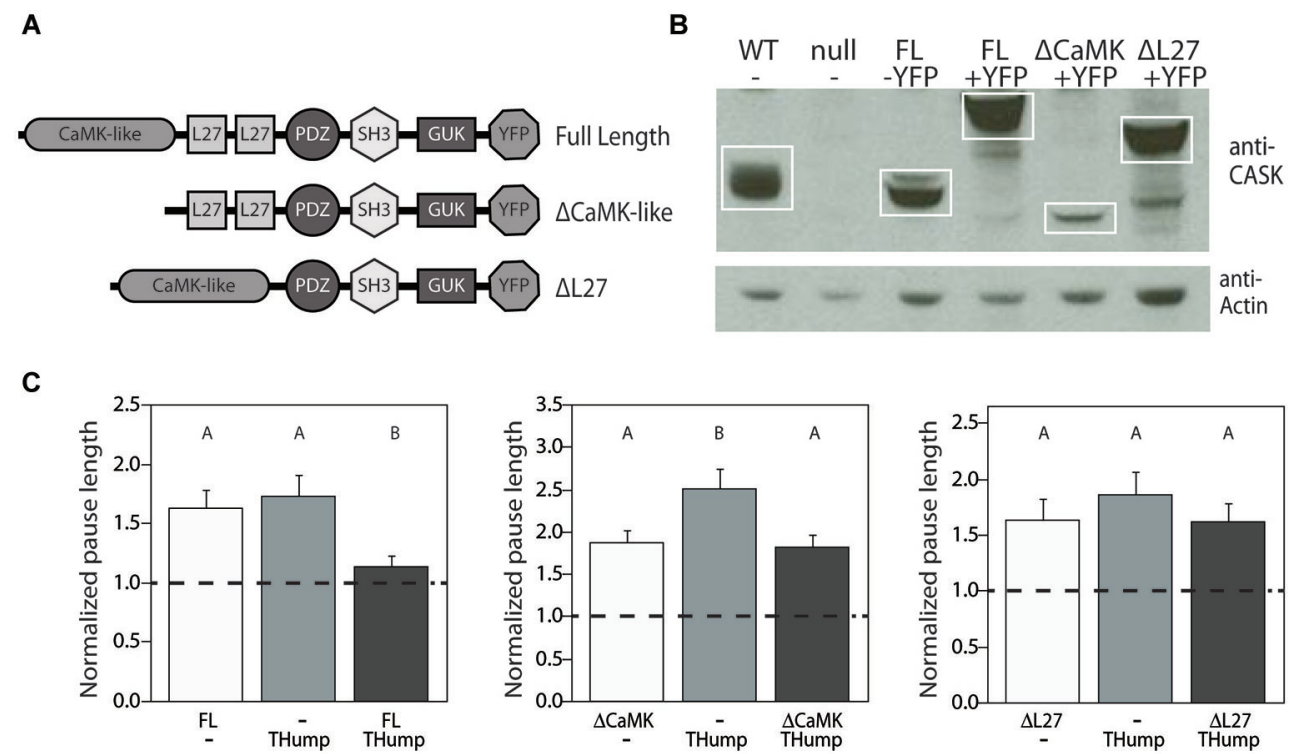

FIGURE 4 | N-terminal domains of CASK- $\beta$ are required for behavioral rescue in dopaminergic cells. (A) Domain structures of CASK- $\beta$ proteins used for rescue. In-frame deletions were generated in the UAS-CASK- $\beta$-YFP construct to ablate either the CaMK-like or L27 domains. (B) CASK- $\beta$-YFP proteins were expressed using a pan-neuronal driver line (C155-Ga/4) and visualized via immunoblot of fly heads. Actin was immunoblotted as a loading control. (C) Expression of full length CASK- $\beta$-YFP with THump-Gal4 rescued pause length, while both domain-truncated CASK- $\beta$ proteins failed to rescue the locomotor deficit when expressed under control of this driver. Data from all groups are normalized to WT control levels which are shown as a dotted black line at 1.0 on the Y-axis of each graph. The magnitude of the deviation from the dotted line is proportional to severity of phenotype. Transgenes present in each group of animals are indicated below the histogram bars: " $\mathrm{FL}$ " $=$ Full Length CASK- $\beta$, " $\Delta$ CaMK" = CaMK-like domain-ablated CASK- $\beta$, and $" \Delta \mathrm{L} 27 "=\mathrm{L} 27$ domain-ablated CASK $-\beta$. All transgenes were on a CASK- $\beta$ mutant background, and letters indicate significant differences between groups $(P<0.05$, ANOVA with Tukey HSD). See Section Methods for details on statistical tests. binding partners for CASK- $\beta$. We found that while CASK may participate in a physical complex with neurexin in some neuronal cell-types, this interaction was not observed in dopaminergic neurons (Mukherjee et al., 2014), suggesting the possibility that there might be other mechanisms by which CASK can regulate vesicle fusion.

One candidate that was present in CASK- $\beta$ pull downs from dopaminergic neurons was Hsc70-4 (also known as HSC4). At the larval neuromuscular junction, $\mathrm{Hsc} 70-4$ has been shown to be required for the coupling of activity to neurotransmitter release (Bronk et al., 2001). The exocytosis phenotype of the Hsc704 mutant at this synapse can be rescued by enhancing calcium influx, which is similar to our finding that driving activity in dopaminergic neurons is able to rescue the CASK- $\beta$ locomotor initiation defect.

To test the idea that CASK and Hsc70-4 might cooperate to regulate locomotor initiation, we examined the genetic interactions between $C A S K-\beta$ and $H s c 70-4$. We first looked at behavior in transheterozygotes of $C A S K-\beta^{P 18}$ (a null allele) and Hsc70-4 $\Delta^{19}$ (a strong hypomorph). As previously reported, CASK- $\beta$ null mutants show gene dose-dependent deficits in multiple locomotor parameters. Interestingly, $\mathrm{Hsc70-4}$ heterozygotes are only defective in "Pause Length", a metric for motor initiation (Figure 5A) but were normal for other parameters, suggesting that Hsc70-4 has a specific role in motor initiation. Homozygotes of this genotype did not survive to adulthood and therefore could not be assayed. Transheterozygotes, animals missing one copy of the CASK- $\beta$ gene and one copy of Hsc70-4, showed a synthetic phenotype worse than either heterozygote alone for motor initiation. In the case of the other parameters, the transheterozygote behaved more similarly to the CASK- $\beta$ heterozygote (Figure 5A), suggesting no synthetic effect and little contribution to phenotype from loss of a single copy of Hsc70-4.

These data were consistent with an interaction of CASK- $\beta$ and Hsc70-4 regulating dopamine release, but since the effects of germline mutations are not limited to dopaminergic neurons, the motor initiation phenotype of the Hsc70-4 mutant could be the result of actions of this gene during development and/or in non-dopaminergic neurons. To test the temporal and spatial specificity of the interaction, we utilized a temperature-sensitive GAL80 (McGuire et al., 2004) to limit THump-Gal4-driven dominant negative Hsc70-4 (Elefant and Palter, 1999) to adult neurons. Expression of dominant negative Hsc70-4 only in adult dopaminergic cells produced a significant motor initiation phenotype but did not affect other locomotor parameters (Figure 5B). These data are supportive of an acute role for Hsc70-4 in regulation of dopaminergic neuron function.

\section{DISCUSSION \\ CASK- $\beta$ REGULATES INITIATION OF MOVEMENT BY AFFECTING THE FUNCTION OF DOPAMINERGIC NEURONS}

The ability to initiate locomotion is critical to an organism's ability to perform other more complex behavioral tasks necessary for survival. As such, the mechanisms involved in generating and 

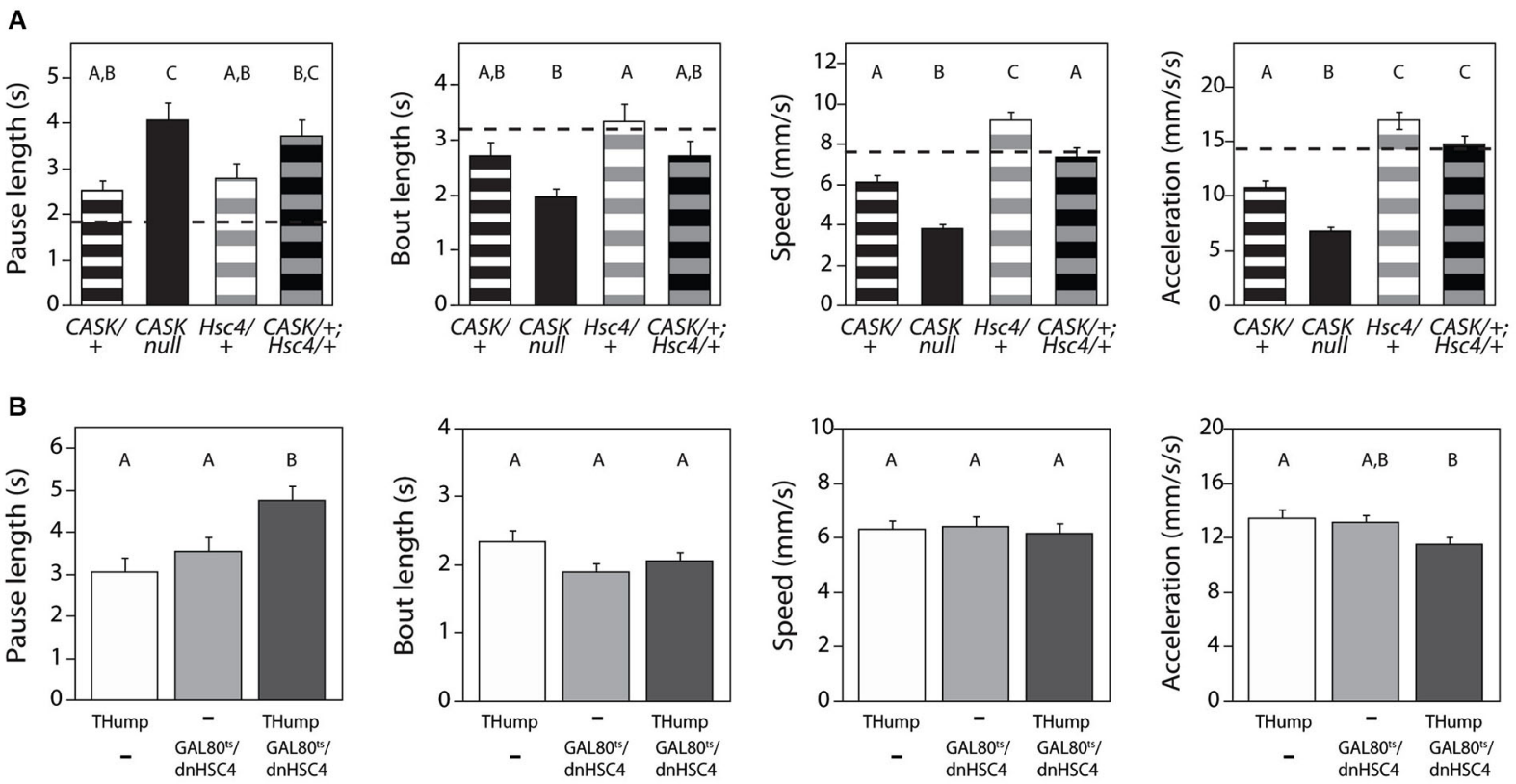

FIGURE 5 | CASK- $\boldsymbol{\beta}$ interacts behaviorally with $\boldsymbol{H s c 7 0 - 4}$. (A) Flies heterozygous for a hypomorphic $\mathrm{Hsc70}-4$ mutation $(\mathrm{Hsc} 4 /+$ ) show a defect in the locomotor pause length parameter similar to that of CASK- $\beta /+$ flies, but less severe than CASK- $\beta$ homozygous null flies. Pause length is significantly worse in transheterozygote flies containing one mutant allele of each gene (CASK- $\beta /+; H s c 4 /+)$, indicating that the two genes likely interact in regulating motor initiation. Hsc70-4/+ flies do not on their own present a phenotype in the other three measured parameters of locomotion, nor do they appear to worsen these parameters when combined with the $C A S K-\beta$ mutation. Here the behavioral performance of WT flies is presented as a dotted black line, with the magnitude of the deviation from the dotted line proportional to

maintaining proper motor initiation are regulated at many different biological levels. CASK has clearly been shown to have a role in governing these processes (Martin and Ollo, 1996; Sun et al., 2009; Slawson et al., 2011). In this study we demonstrate that one aspect of locomotor behavior, initiation of movement, is dependent on the presence of CASK- $\beta$ in dopaminergic neurons. Interestingly, other facets of the CASK- $\beta$ null locomotor phenotype were not rescued by expression of CASK- $\beta$ in this subset of neurons, but required much broader expression of CASK- $\beta$ (Slawson et al., 2011). The finding that there is independent control of functional locomotor parameters is quite novel. Future studies involving cell-specific rescue of these parameters could lead to identification of new motor circuit elements. Since CASK- $\beta$ has been shown to interact with unique repertoires of proteins across different cell types (Mukherjee et al., 2014), our results suggest the possibility that the complex and multi-faceted behavioral phenotype of the null mutant is due to distinct molecular complexes being disrupted in these different groups of cells.

\section{A NOVEL ROLE FOR CASK- $\beta$ IN REGULATION OF NEUROTRANSMITTER RELEASE IN ADULT DOPAMINERGIC NEURONS}

While loss of the entire CASK gene can disrupt evoked release at both larval and adult neuromuscular junctions (Zordan et al., severity of phenotype. Genotypes of each group of animals are indicated below the histogram bars: "CASK" $=C A S K-\beta^{\mathrm{P} 18}$, a null allele; " HsC4" = Hsc70-4 $\Delta^{19}$, a hypomorphic allele; " + " = a WT allele. (B) A dominant negative Hsc70-4 transgene was expressed under control of THump-Gal4 and expression limited to adulthood using tub-Gal80ts. Here "Thump" = THump-Gal4 and "Gal80ts/dnHSC4" = tub-Gal80ts/UAS-[dn]Hsc70-4. Expression of this dominant negative construct in adult dopaminergic cells results in reduced motor initiation behavior (as evidenced by the change in the pause length parameter), but no significant changes in the other indices of locomotor behavior. As before, letters indicate significant differences between groups ( $P<0.05$, ANOVA with Tukey HSD). See Section Methods for details on statistical tests.
2005; Sun et al., 2009; Chen and Featherstone, 2011), there has been less work on the role of CASK at adult central synapses. A study on the role of CASK- $\beta$ in learning (Malik et al., 2013) demonstrated a decrease in calcium responsiveness of the mutant in mushroom bodies. Here we show that in CASK- $\beta$ mutants, vesicle fusion induced by a cholinergic agonist is reduced in dopaminergic neurons. These data suggest a model in which the defect in motor initiation is due to abnormal coupling of neuronal activity with neurotransmitter release. Consistent with this hypothesis, driving activity at very high levels in dopaminergic neurons with dTRPA1 appears to rescue initiation without affecting other locomotor parameters in CASK- $\beta$ mutants. This argues for a function role of CASK- $\beta$ in dopamine release.

Neurexin was a likely candidate partner for CASK- $\beta$ as a regulator of neurotransmitter release (Hata et al., 1996; Mukherjee et al., 2008; Sun et al., 2009). Previous work had profiled cell-specific CASK- $\beta$ protein complexes by identifying proteins present in immunoprecipitations of tagged CASK- $\beta$ expressed in particular cell types on a null mutant genetic background (Mukherjee et al., 2014). In that study, neurexin was identified as a binding partner in pull-downs when tagged CASK- $\beta$ was driven in all neurons, but when the pull-downs were repeated from dopaminergic neurons, neurexin was not present. There 
were, however, a number of other proteins known to be involved in neurotransmitter release which were enriched in dopaminergic neurons relative to other populations, including Comatose (the Drosophila NSF homolog), Synapsin, and Hsc70-4. The presence of Synapsin was reduced in pull-downs using tagged versions of CASK- $\beta$ lacking either the CaMK or L27 domain, suggesting that this might be a critical interaction, since CASK- $\beta$ lacking these domains fails to rescue motor initiation.

The most potentially interesting candidate from the list of interacting proteins, however, was Hsc70-4. At the Drosophila third instar larval neuromuscular junction, this protein has been shown to modulate the coupling of neuronal activity and calcium to neurotransmitter release (Bronk et al., 2001). Defects in neurotransmitter release in hypomorphic $\mathrm{Hsc70-4}$ mutants can be rescued by increasing extracellular calcium or by high frequency stimulation, which transiently increases resting calcium in the presynaptic terminal. This was very reminiscent of our results (Figure 3) demonstrating that driving activity in dopaminergic neurons with dTRPA1 can rescue the locomotor initiation phenotype of $C A S K-\beta$ mutants. This similarity suggested that the interaction with Hsc70-4 might be functionally important for the $C A S K-\beta$ null phenotype. Genetic interactions between CASK$\beta$ and both $H s c 70-4$ mutants and a dominant negative Hsc70-4 transgene strongly supported this hypothesis.

There was a surprising amount of functional specificity for dopaminergic neurons in this interaction given that the protein association between CASK and Hsc70-4 was seen in multiple neuron types (Mukherjee et al., 2014). Hsc70-4/+ heterozygous mutants had defects only in the initiation parameter of locomotion, but not in the other locomotor metrics (Figure 5). These other parameters are controlled by independent circuits that involve non-dopaminergic neurons. While it seems likely that the calcium responses in these circuits are also blunted in the CASK$\beta$ mutant (Malik et al., 2013), their dysfunction does not seem to involve an Hsc70-4-sensitive pathway since reduction of gene copy number has no effect on them and did not enhance their $C A S K-\beta /+$ phenotype. This could indicate that they have other non-Hsc70-4-dependent mechanisms for coupling release to calcium or that the disruption of the function of these circuits in the CASK- $\beta$ mutant does not involve changes in neurotransmitter release.

The molecular details of how Hsc70-4 and CASK- $\beta$ regulate the vesicle fusion machinery remain to be elucidated. There are many possibilities, several of which involve changes in localization of release-regulating proteins to enhance their access to calcium. The mechanism is likely to be complex, but our data indicate it may have special relevance in dopaminergic neurons.

\section{OTHER ROLES FOR CASK- $\beta$ IN DOPAMINERGIC NEURONS?}

Since CASK has many binding partners, it is possible that this protein does more in dopaminergic neurons than just regulate neurotransmitter release. It has been recently reported that this protein also associates with mitochondria (Mukherjee et al., 2014), which are a known site of dysfunction in Parkinson's Disease. Analysis of CASK- $\beta$ binding partners (Mukherjee et al., 2014) also identified Dj1- $\beta$, a recessive cause of familial Parkinson's disease in humans, as a dopaminergic neuron-specific binding partner. Investigation of the role of CASK in age-related aspects of dopaminergic neuron function may provide some insights into the specific cell biology of these neurons.

\section{ACKNOWLEDGMENTS}

This work was funded by NIH grants R01 GM54408 (to Leslie C. Griffith) and F31 NS064679 (to Justin B. Slawson). The Brandeis Imaging Facility was supported by NIH grant P30 NS045713. We would like to thank Bethany Christmann for brain dissection support and Ed Dougherty for imaging assistance. We would also like to thank the curators of Flybase and the Bloomington Drosophila Stock Center for providing resources critical for the completion of this work.

\section{SUPPLEMENTARY MATERIAL}

The Supplementary Material for this article can be found online at: http://www.frontiersin.org/journal/10.3389/fnbeh.2014. 00394/abstract

Figure S1 | TH-Gal4 and THump-Gal4 express in all dopaminergic neurons in all adult brain dopaminergic clusters. (A) The expression pattern of $T H-G a l 4$ was assessed by crossing it to UAS-mCD8GFP. Brains from these animals were dissected, fixed, stained with anti-TH and anti-GFP, and visualized using confocal imaging. Cell counts demonstrate that all TH+ cells in the PPL1, PPL2, PPM1/2, PPM3, $\mathrm{PAL}$, and SOG clusters were present in the $\mathrm{TH}-\mathrm{Ga} / 4$ expression pattern, since virtually all TH+ cells were also GFP+. (B) The same approach was used to map dopaminergic cells within the THump-Gal4 expression pattern. Like TH-Gal4, THump-Gal4 also expresses in all $\mathrm{TH}+$ cells.

Figure S2 | Loss of dopaminergic cells from the C164-Gal4 expression pattern blocks rescue. Behavioral rescue experiments using C164-Gal4 to drive ectopic expression of CASK- $\beta$ in the central nervous system was repeated as in Slawson et al. (2011). In this instance, activity of the GAL4 driver in dopaminergic cells was repressed by the presence of $\mathrm{TH}-\mathrm{Ga} / 80$. Preventing expression of CASK- $\beta$ in these cells prevented the behavioral rescue of pause length, further implicating these cells as the cellular locus for CASK- $\beta$ in motor behavior. "CASK" = UAS-CASK- $\beta$, "THGal80" = TH-Gal80, and "C164" = C164-Gal4. Data from all groups are normalized to WT control levels, which are shown as a dotted black line at 1.0 on the Y-axis of each graph. The magnitude of the deviation from the dotted line is proportional to severity of phenotype. All transgenes were on a CASK- $\beta$ mutant background, and letters indicate significant differences between groups $(P<0.05$, ANOVA with Tukey HSD). See Section Methods for details on statistical tests.

\section{REFERENCES}

Alvarez-Erviti, L., Rodriguez-Oroz, M. C., Cooper, J. M., Caballero, C., Ferrer, I., Obeso, J. A., et al. (2010). Chaperone-mediated autophagy markers in Parkinson disease brains. Arch. Neurol. 67, 1464-1472. doi: 10.1001/archneurol.2010. 198

Atasoy, D., Schoch, S., Ho, A., Nadasy, K. A., Liu, X., Zhang, W., et al. (2007). Deletion of CASK in mice is lethal and impairs synaptic function. Proc. Natl. Acad. Sci. U S A 104, 2525-2530. doi: 10.1073/pnas.0611003104

Auluck, P. K., Chan, H. Y., Trojanowski, J. Q., Lee, V. M., and Bonini, N. M. (2002). Chaperone suppression of alpha-synuclein toxicity in a Drosophila model for Parkinson's disease. Science 295, 865-868. doi: 10.1126/science.10 67389 
Braak, H., and Del Tredici, K. (2008). Invited article: nervous system pathology in sporadic Parkinson disease. Neurology 70, 1916-1925. doi: 10.1212/01.wnl. 0000312279.49272.9f

Brand, A. H., and Perrimon, N. (1993). Targeted gene expression as a means of altering cell fates and generating dominant phenotypes. Development 118, 401-415.

Bronk, P., Wenniger, J. J., Dawson-Scully, K., Guo, X., Hong, S., Atwood, H. L., et al. (2001). Drosophila Hsc70-4 is critical for neurotransmitter exocytosis in vivo. Neuron 30, 475-488. doi: 10.1016/s0896-6273(01)00292-6

Butz, S., Okamoto, M., and Südhof, T. C. (1998). A tripartite protein complex with the potential to couple synaptic vesicle exocytosis to cell adhesion in brain. Cell 94, 773-782. doi: 10.1016/s0092-8674(00)81736-5

Chen, K., and Featherstone, D. E. (2011). Pre and postsynaptic roles for Drosophila CASK. Mol. Cell. Neurosci. 48, 171-182. doi: 10.1016/j.mcn.2011.07.009

Costa, R. M. (2007). Plastic corticostriatal circuits for action learning: what's dopamine got to do with it? Ann. N Y Acad. Sci. 1104, 172-191. doi: 10. 1196/annals.1390.015

Edelstein, A., Amodaj, N., Hoover, K., Vale, R., and Stuurman, N. (2010). Computer control of microscopes using $\mu$ Manager. Curr. Protoc. Mol. Biol. 20, 14.20.1-14.20.17. doi: 10.1002/0471142727.mb1420s92

Elefant, F., and Palter, K. B. (1999). Tissue-specific expression of dominant negative mutant Drosophila HSC70 causes developmental defects and lethality. Mol. Biol. Cell 10, 2101-2117. doi: 10.1091/mbc.10.7.2101

Feany, M. B., and Bender, W. W. (2000). A Drosophila model of Parkinson's disease. Nature 404, 394-398. doi: 10.1038/35006074

Fischer, J. A., Giniger, E., Maniatis, T., and Ptashne, M. (1988). GAL4 activates transcription in Drosophila. Nature 332, 853-856. doi: 10.1038/332853a0

Friggi-Grelin, F., Coulom, H., Meller, M., Gomez, D., Hirsh, J., and Birman, S. (2003). Targeted gene expression in Drosophila dopaminergic cells using regulatory sequences from tyrosine hydroxylase. J. Neurobiol. 54, 618-627. doi: $10.1002 /$ neu. 10185

Gillespie, J. M., and Hodge, J. J. (2013). CASK regulates CaMKII autophosphorylation in neuronal growth, calcium signaling and learning. Front. Mol. Neurosci. 6:27. doi: 10.3389/fnmol.2013.00027

Greenspan, R. J. (1997). Fly Pushing: The Theory and Practice of Drosophila Genetics. Cold Spring Harbor, NY: Cold Spring Harbor Press.

Hamada, F. N., Rosenzweig, M., Kang, K., Pulver, S. R., Ghezzi, A., Jegla, T. J., et al. (2008). An internal thermal sensor controlling temperature preference in Drosophila. Nature 454, 217-220. doi: 10.1038/nature07001

Hata, Y., Butz, S., and Südhof, T. C. (1996). CASK: a novel dlg/PSD95 homolog with an $\mathrm{N}$-terminal calmodulin-dependent protein kinase domain identified by interaction with neurexins. J. Neurosci. 16, 2488-2494.

Kong, E. C., Woo, K., Li, H., Lebestky, T., Mayer, N., Sniffen, M. R., et al. (2010). A pair of dopamine neurons target the D1-like dopamine receptor DopR in the central complex to promote ethanol-stimulated locomotion in Drosophila. PLoS One 5:e9954. doi: 10.1371/journal.pone.0009954

Leech, C. A., and Sattelle, D. B. (1993). Acetylcholine receptor/channel molecules of insects. EXS 63, 81-97. doi: 10.1007/978-3-0348-7265-2_5

Lees, A. J., Hardy, J., and Revesz, T. (2009). Parkinson's disease. Lancet 373, 20552066. doi: 10.1016/S0140-6736(09)60492-X

Malik, B. R., Gillespie, J. M., and Hodge, J. J. (2013). CASK and CaMKII function in the mushroom body alpha'/beta' neurons during Drosophila memory formation. Front. Neural Circuits 7:52. doi: 10.3389/fncir.2013.00052

Mao, Z., and Davis, R. L. (2009). Eight different types of dopaminergic neurons innervate the Drosophila mushroom body neuropil: anatomical and physiological heterogeneity. Front. Neural Circuits 3:5. doi: 10.3389/neuro.04.005.2009

Marble, D. D., Hegle, A. P., Snyder, E. D. 2nd, Dimitratos, S., Bryant, P. J., and Wilson, G. F. (2005). Camguk/CASK enhances Ether-á-go-go potassium current by a phosphorylation-dependent mechanism. J. Neurosci. 25, 48984907. doi: 10.1523/jneurosci.4566-04.2005

Martin, J. R., Ernst, R., and Heisenberg, M. (1998). Mushroom bodies suppress locomotor activity in Drosophila melanogaster. Learn. Mem. 5, 179-191.

Martin, J. R., and Ollo, R. (1996). A new Drosophila Ca2+/calmodulin-dependent protein kinase (Caki) is localized in the central nervous system and implicated in walking speed. EMBO J. 15, 1865-1876.

Martin, J. R., Raabe, T., and Heisenberg, M. (1999). Central complex substructures are required for the maintenance of locomotor activity in Drosophila melanogaster. J. Comp. Physiol. A 185, 277-288. doi: 10.1007/s003590050387
McGuire, S. E., Mao, Z., and Davis, R. L. (2004). Spatiotemporal gene expression targeting with the TARGET and gene-switch systems in Drosophila. Sci. STKE 2004:pl6. doi: 10.1126/stke.2202004pl6

Miesenböck, G., De Angelis, D. A., and Rothman, J. E. (1998). Visualizing secretion and synaptic transmission with $\mathrm{pH}$-sensitive green fluorescent proteins. Nature 394, 192-195. doi: 10.1038/28190

Missler, M., and Sudhof, T. C. (1998). Neurexins: three genes and 1001 products. Trends Genet. 14, 20-26. doi: 10.1016/s0168-9525(97)01324-3

Mukherjee, K., Sharma, M., Urlaub, H., Bourenkov, G. P., Jahn, R., Südhof, T. C., et al. (2008). CASK functions as a Mg2+-independent neurexin kinase. Cell 133, 328-339. doi: 10.1016/j.cell.2008.02.036

Mukherjee, K., Slawson, J. B., Christmann, B. L., and Griffith, L. C. (2014). Neuron-specific protein interactions of Drosophila CASK- $\beta$ are revealed by mass spectrometry. Front. Mol. Neurosci. 7:58. doi: 10.3389/fnmol.2014. 00058

Muñoz-Soriano, V., and Paricio, N. (2011). Drosophila models of Parkinson's disease: discovering relevant pathways and novel therapeutic strategies. Parkinsons Dis. 2011:520640. doi: 10.4061/2011/520640

Neckameyer, W. S. (1998). Dopamine and mushroom bodies in Drosophila: experience-dependent and -independent aspects of sexual behavior. Learn. Mem. 5, 157-165.

Ng, M., Roorda, R. D., Lima, S. Q., Zemelman, B. V., Morcillo, P., and Miesenböck, G. (2002). Transmission of olfactory information between three populations of neurons in the antennal lobe of the fly. Neuron 36, 463-474. doi: 10.1016/s08966273(02)00975-3

Obeso, J. A., Rodriguez-Oroz, M. C., Goetz, C. G., Marin, C., Kordower, J. H., Rodriguez, M., et al. (2010). Missing pieces in the Parkinson's disease puzzle. Nat. Med. 16, 653-661. doi: 10.1038/nm.2165

Palmiter, R. D. (2008). Dopamine signaling in the dorsal striatum is essential for motivated behaviors: lessons from dopamine-deficient mice. Ann. N Y Acad. Sci. 1129, 35-46. doi: 10.1196/annals.1417.003

Pemberton, S., Madiona, K., Pieri, L., Kabani, M., Bousset, L., and Melki, R. (2011). Hsc70 protein interaction with soluble and fibrillar alpha-synuclein. J. Biol. Chem. 286, 34690-34699. doi: 10.1074/jbc.m111.261321

Phelps, C. B., and Brand, A. H. (1998). Ectopic gene expression in Drosophila using GAL4 system. Methods 14, 367-379. doi: 10.1006/meth.1998.0592

Piccini, P. (2004). Neurodegenerative movement disorders: the contribution of functional imaging. Curr. Opin. Neurol. 17, 459-466. doi: 10.1097/01.wco. $0000137538.84115 .3 \mathrm{c}$

Riemensperger, T., Isabel, G., Coulom, H., Neuser, K., Seugnet, L., Kume, K., et al. (2011). Behavioral consequences of dopamine deficiency in the Drosophila central nervous system. Proc. Natl. Acad. Sci. U S A 108, 834-839. doi: 10. 1073/pnas.1010930108

Sala, G., Stefanoni, G., Arosio, A., Riva, C., Melchionda, L., Saracchi, E., et al. (2014). Reduced expression of the chaperone-mediated autophagy carrier hsc70 protein in lymphomonocytes of patients with Parkinson's disease. Brain Res. 1546, 46-52. doi: 10.1016/j.brainres.2013.12.017

Schapira, A. H. (2009). Etiology and pathogenesis of Parkinson disease. Neurol. Clin. 27, 583-603. doi: 10.1016/j.ncl.2009.04.004

Slawson, J. B., Kim, E. Z., and Griffith, L. C. (2009). High-resolution video tracking of locomotion in adult Drosophila melanogaster. J. Vis. Exp. doi: 10.3791/1096

Slawson, J. B., Kuklin, E. A., Ejima, A., Mukherjee, K., Ostrovsky, L., and Griffith, L. C. (2011). Central regulation of locomotor behavior of Drosophila melanogaster depends on a CASK isoform containing CaMK-like and L27 domains. Genetics 187, 171-184. doi: 10.1534/genetics.110.123406

Soll, D. R. (1995). The use of computers in understanding how animal cells crawl. Int. Rev. Cytol. 163, 43-104. doi: 10.1016/s0074-7696(08) 62209-3

Soll, D. R., Wessels, D., Voss, E., and Johnson, O. (2001). Computer-assisted systems for the analysis of amoeboid cell motility. Methods Mol. Biol. 161, 45-58. doi: 10. 1385/1-59259-051-9:045

Strauss, R. (2002). The central complex and the genetic dissection of locomotor behaviour. Curr. Opin. Neurobiol. 12, 633-638. doi: 10.1016/s09594388(02)00385-9

Strauss, R., and Heisenberg, M. (1993). A higher control center of locomotor behavior in the Drosophila brain. J. Neurosci. 13, 1852-1861.

Sun, M., Liu, L., Zeng, X., Xu, M., Liu, L., Fang, M., et al. (2009). Genetic interaction between Neurexin and CAKI/CMG is important for synaptic function in 
Drosophila neuromuscular junction. Neurosci. Res. 64, 362-371. doi: 10.1016/j. neures.2009.04.009

Surmeier, D. J., Guzman, J. N., Sanchez-Padilla, J., and Goldberg, J. A. (2010). What causes the death of dopaminergic neurons in Parkinson's disease? Prog. Brain Res. 183, 59-77. doi: 10.1016/s0079-6123(10)83004-3

Thomas, B., and Beal, M. F. (2007). Parkinson's disease. Hum. Mol. Genet. 16, R183R194. doi: $10.1093 / \mathrm{hmg} / \mathrm{ddm} 159$

Trinh, K., Moore, K., Wes, P. D., Muchowski, P. J., Dey, J., Andrews, L., et al. (2008). Induction of the phase II detoxification pathway suppresses neuron loss in Drosophila models of Parkinson's disease. J. Neurosci. 28, 465-472. doi: 10. 1523/jneurosci.4778-07.2008

Vaarmann, A., Kovac, S., Holmström, K. M., Gandhi, S., and Abramov, A. Y. (2013). Dopamine protects neurons against glutamate-induced excitotoxicity. Cell Death Dis. 4:e455. doi: 10.1038/cddis.2012.194

Wang, J. W., Wong, A. M., Flores, J., Vosshall, L. B., and Axel, R. (2003). Two-photon calcium imaging reveals an odor-evoked map of activity in the fly brain. Cell 112, 271-282. doi: 10.1016/s0092-8674(03)00004-7

White, K. E., Humphrey, D. M., and Hirth, F. (2010). The dopaminergic system in the aging brain of Drosophila. Front. Neurosci. 4:205. doi: 10.3389/fnins.2010. 00205

Whitworth, A. J., Theodore, D. A., Greene, J. C., Benes, H., Wes, P. D., and Pallanck, L. J. (2005). Increased glutathione S-transferase activity rescues dopaminergic neuron loss in a Drosophila model of Parkinson's disease. Proc. Natl. Acad. Sci. U S A 102, 8024-8029. doi: 10.1073/pnas.05010 78102
Zheng, C. Y., Seabold, G. K., Horak, M., and Petralia, R. S. (2011). MAGUKs, synaptic development and synaptic plasticity. Neuroscientist 17, 493-512. doi: 10. $1177 / 1073858410386384$

Zordan, M. A., Massironi, M., Ducato, M. G., Te Kronnie, G., Costa, R., Reggiani, C., et al. (2005). Drosophila CAKI/CMG protein, a homolog of human CASK, is essential for regulation of neurotransmitter vesicle release. J. Neurophysiol. 94, 1074-1083. doi: 10.1152/jn.00954.2004

Conflict of Interest Statement: The authors declare that the research was conducted in the absence of any commercial or financial relationships that could be construed as a potential conflict of interest.

Received: 06 September 2014; accepted: 25 October 2014; published online: 18 November 2014.

Citation: Slawson JB, Kuklin EA, Mukherjee K, Pírez N, Donelson NC and Griffith LC (2014) Regulation of dopamine release by CASK- $\beta$ modulates locomotor initiation in Drosophila melanogaster. Front. Behav. Neurosci. 8:394. doi: 10.3389/fnbeh.2014.00394

This article was submitted to the journal Frontiers in Behavioral Neuroscience. Copyright (๑) 2014 Slawson, Kuklin, Mukherjee, Pirez, Donelson and Griffith. This is an open-access article distributed under the terms of the Creative Commons Attribution License (CC BY). The use, distribution and reproduction in other forums is permitted, provided the original author(s) or licensor are credited and that the original publication in this journal is cited, in accordance with accepted academic practice. No use, distribution or reproduction is permitted which does not comply with these terms. 